\title{
Factors Influencing Practice of Optimal Dietary Intake of Alcoholics under Rehabilitation in Asumbi-Homabay, Kenya
}

\section{Lucy Amanya Mutuli ${ }^{*}$ Peter Bukhala and Gordon Nguka}

Masinde Muliro University of Science and Technology, Kakamega, Kenya

*Corresponding author: Lucy Amanya Mutuli, Masinde Muliro University of Science and Technology, Kakamega, Kenya, Tel: +46 31 7864694; E-mail: amanyalucy@yahoo.com

Received date: October 14, 2017, Accepted date: October 17, 2017, Published date: October 20, 2017

Copyright: (C) 2017 Mutuli LA, et al. This is an open-access article distributed under the terms of the Creative Commons Attribution License, which permits unrestricted use, distribution, and reproduction in any medium, provided the original author and source are credited.

\begin{abstract}
Background: Optimal dietary intake in alcohol rehabilitation is a pathway that reflects self-care, commitment to staying sober and is a vital adjunct in effective alcohol rehabilitation.
\end{abstract}

Objective: This qualitative study sought to elicit facilitators and barriers that influence the practice of optimal dietary intake during the three months of alcohol rehabilitation.

Methods: A question guide was utilized to solicit information through in-depth interviews from 15 alcoholics and 5 health professionals until saturation point. Constant comparative approach was used to analyze verbatim transcripts obtained from in-depth interviews. This analysis entailed three stages including open, axial and selective coding.

Results: Recuperating alcoholics' appreciated the health benefits of practicing optimal dietary intake in restoring their health, with health professionals being significantly supportive which translated into increased self-efficacy to practice of optimal dietary intake during alcohol rehabilitation.

Conclusions: The rehabilitation centres should have nutritionists besides other health professionals who can offer individualized nutrition services needed by the recuperating alcoholics.

Keywords: Alcoholism; Dietary intake; Rehabilitation; Psychosocial factors

\section{Introduction}

Alcoholism is a chronic relapsing disorder which alters brain functioning causing impaired judgment, reduced self-control, memory and learning functions [1]. These brain changes causes' severe biochemical imbalances which renders the body more solely dependent on alcohol to make it feel stable and to alleviate the alcohol cravings [2]. Optimal dietary intake restores the physiological disruptions of the body using specific nutrients which the brain is wired on that affects how biochemical imbalances are restored and alcohol cravings are regulated [1]. Optimal dietary intake is a lifestyle treatment strategy that is aimed to help the alcoholic maintain sobriety.

In Kenya alcoholic treatment remains expensive, especially for inpatients who are rehabilitated for three months before discharge. However, the outcome of alcohol treatment reveals uncertainties through the relapse ranges of $60 \%$ to $90 \%$ after treatment $[3,4]$. Evidence shows that the emphasis on optimal dietary intake is very limited in these rehabilitation centers $[5,6]$. Yet these are strategies that can foster healthy lifestyle changes that contribute to long-term maintenance of recovery from alcohol. This setback is associated with sessions that have no input from the alcoholics but simply impose an idea that leaves the alcoholic with mixed perceptions regarding optimal dietary intake in effectively treating alcoholism and reducing relapse [3].
Given that, health professionals of the rehabilitation centers devise and transmit optimal dietary intake information without considering alcoholics' cognitive and experiential aspects such as attitude, social perceptions, and self-efficacy and control beliefs [7]. These psychosocial attributes are significant in explaining how an alcoholic makes the decision or arrives at intention to practice optimal dietary intake during alcohol rehabilitation [8]. For instance, an alcoholics' subjective likelihood about the likely outcomes of the optimal dietary intake determines their attitude towards it.

Moreover, alcoholics attempting to practice optimal dietary intake often fail as they have to comply with social expectations and encounter obstacles that may facilitate or impede their practice. An alcoholics' decision to practice optimal dietary intake is determined by a combination of psychosocial factors which need to be identified and assessed. These psychosocial factors have been identified in a number of cognitive theories and in this context; Theory of Planned Behaviour.

This study intended to use the Theory of Planned Behaviour concepts'; attitude, subjective norm and perceived behavioural control as determinants of the practising of optimal dietary intake during alcohol rehabilitation. The researchers sought to establish the main themes underlying each psychosocial factor that influenced practise of optimal dietary intake during rehabilitation.

These results were more suggestive than affirmative limiting the possibility for quantitative comparisons but significant in development of an instrument for quantitative section. 


\section{Methods}

\section{Study area, design and population}

Asumbi-Homabay located in Homabay County, Nyanza region of Kenya formed the study area mainly because of the existence of Asumbi rehabilitation center. This center was purposively sampled with the target that it receives numerous alcoholic patients both males and females from different parts of the country, offers standardized rehabilitation services to alcoholic rehabilitees and it's accredited by NACADA. This cross-sectional study was conducted between January and March, 2016 at Asumbi rehabilitation center. Sample criteria included female and male alcoholics aged 15-65 years who were admitted not more than a week prior to commencement of the data collection and those who voluntarily consented to participate in the study.

Alcoholics with active psychotic symptoms, addicts of substances other than alcohol and not intending to complete the three months of rehabilitation in Asumbi center were excluded from the study. A purposive sampling technique was used to select 20 out of 50 participants based on the inclusion criteria for this study, recommendations on qualitative research and utility of the TPB, and limitation of resources. The participants were elucidated for the purpose of the study before their participation in the in-depth interviews. Ethical approval was given by National Council for Science and Technology. Research authorization was granted by the National Agency for the Campaign against Drug Abuse. We sought informed consent from the respondents who were informed on the research procedures, details and assured of confidentiality.

\section{Study instrument}

An in-depth interview was conducted using a question guide developed based on Ajzen's theoretical framework. The main theme explored during the in-depth interview was psychosocial factors affecting practice of optimal dietary intake during alcohol rehabilitation. A total of 8 health professionals who were engaged in rehabilitating alcoholics at Asumbi Center were purposively selected and interviewed, the findings of these interviews were used to construct a question guide for the study. Two nutrition experts and a researcher then critically examined, discussed and provided inputs into the content of the question guide; they also assisted in refining it.

The question guide was then pretested on a sample of 10 alcoholics under rehabilitation at Asumbi Center who were randomly selected. The question guide was utilized to solicit information through indepth interviews in groups consisting 15 alcoholics and 5 health professionals until saturation point. Verbatim transcripts were prepared regarding the factors these alcoholics encountered during their practice of optimal dietary intake while on rehabilitation. The procedure of preparing verbatim transcripts was essential to determine the salient consequences, referents and circumstances that form the belief structure underlying the intention to practice optimal dietary intake while on alcohol rehabilitation.

\section{Data analysis}

Constant comparative approach grounded in the Theory Planned Behaviour was used to analyze verbatim transcripts obtained from indepth interviews. This analysis entailed three stages including open, axial and selective coding. In the open coding phase, verbatim transcripts generated during the in-depth interviews were examined and updated by listening to tapes to identify outstanding categories of information. In axial phase, the inductive coding categories were saturated and a set of categories developed where we identified several categories from the open coding list that were compared across to explore themes. The central phenomenon of interest was positioned at the centre of the theory and linked with other categories. In the selective phase, the inductive coding obtained was then organized into a coding paradigm that presents a theoretical model of the process under investigation.

\section{Results}

The interview responses provided a detailed source of information about behavioral beliefs, normative beliefs and control beliefs. Constant comparative approach analysis identified familiar verbatim transcripts on salient consequences (advantages/disadvantages), social referents (approval/disapproval) and circumstances (facilitator/ barrier).

\section{Behavioural beliefs}

Participants were aware that to attain optimal dietary intake during alcohol rehabilitation they have to eat a balanced diet from variety of foods to achieve their diet adequacy. The importance of having a balanced diet included 'restoring organs affected by alcohol' 17 (87\%) thus providing a 'state of wellbeing' 10 (50\%). Diet adequacy defined as consumption of a meal that is satisfactory to an individual alcoholic was also recognized as a component for optimal dietary intake with the following reported benefits.

Basically, 7 (37\%) reported that diet adequacy, 'helps to prevent muscle wasting, thus 'help to achieve metabolic demands' and 5 (25\%) argued that it 'reduces fatigue. There were also other recognized benefits, such as 'reduces nutrient deficiencies' reported by $10(50 \%)$ and 'ability of diet adequacy to hydrate the body' was stated by 10 (50\%), recognized that it 'improved brain chemistry'. These findings have been summarised in (Table 1).

\begin{tabular}{|l|l|l|}
\hline Factors & Responses percentage (\%) & Frequency (n=20) \\
\hline Advantages & \multicolumn{2}{l|}{} \\
\hline Eating a balanced diet & 50 & 10 \\
\hline Restores tissues affected by alcohol & 75 & 15 \\
\hline Helps in detoxifying from alcohol & 62 & 12 \\
\hline Corrects hepatic encephalopathy & & 12 \\
\hline
\end{tabular}


Citation: Mutuli LA, Bukhala P, Nguka G (2017) Factors Influencing Practice of Optimal Dietary Intake of Alcoholics under Rehabilitation in Asumbi-Homabay, Kenya. J Gen Pract (Los Angel) 5: 334. doi:10.4172/2329-9126.1000334

Page 3 of 7

\begin{tabular}{|c|c|c|}
\hline Corrects neurological disorders & 62 & 12 \\
\hline Restores organs affected by alcohol & 87 & 17 \\
\hline Restores state of wellbeing & 50 & 10 \\
\hline \multicolumn{3}{|l|}{ Eating a variety of foods } \\
\hline Avoids erratic blood sugars & 50 & 10 \\
\hline Stabilizes neurotransmitters & 62 & 12 \\
\hline Maintains mental clarity & 37 & 7 \\
\hline Reduces cravings for calorie dense foods & 62 & 12 \\
\hline \multicolumn{3}{|l|}{ Diet adequacy } \\
\hline Reduces fatigue & 25 & 5 \\
\hline Prevents muscle wasting & 37 & 7 \\
\hline Achieves metabolic demands & 37 & 7 \\
\hline Reduces nutrient deficiencies & 50 & 10 \\
\hline Hydrates the body & 50 & 10 \\
\hline Improves brain chemistry & 37 & 7 \\
\hline \multicolumn{3}{|l|}{ Disadvantages } \\
\hline \multicolumn{3}{|l|}{ Eating a balanced diet } \\
\hline Requires skills in dietetics & 62 & 12 \\
\hline Requires nutrition knowledge & 75 & 15 \\
\hline \multicolumn{3}{|l|}{ Eating a variety of foods } \\
\hline It's expensive to afford & 62 & 12 \\
\hline It needs a lot of time to prepare & 75 & 15 \\
\hline It's not practical & 62 & 12 \\
\hline \multicolumn{3}{|l|}{ Diet adequacy } \\
\hline It's difficult to approximate & 75 & 15 \\
\hline Fills the urge for alcohol intake & 50 & 10 \\
\hline
\end{tabular}

Table 1: Attitudinal beliefs affecting optimal dietary intake in alcohol rehabilitation.

\section{Normative beliefs}

Normative beliefs are the social expectations of important people in alcoholic's life regarding the practice of optimal dietary intake with having a balanced diet from variety of foods and consuming a diet that is adequate. Significant others who encouraged eating of a balanced diet included 'health professional' $15(75 \%)$, 'family members and friends' had a similar influence of $12(62 \%)$, and $10(50 \%)$ of colleagues.

Practically 12 (62\%) of the participants reported 'medical professionals' as people whose opinion was most influential on their decision to consume a diet of varied foods. 'Family members' 12 (62\%) and friends 10 (50\%) were also influencing the alcoholics to have a diet of varied foods. The alcoholic's colleagues 10 (50\%) and the environment $15(75 \%)$ were reported as the disapproving group to eating a variety of foods during alcohol rehabilitation. The decision to attain diet adequacy was supported by medical professionals $10(50 \%)$, family members 12 (62\%), friends 7 (37\%), and colleagues 7 (37\%).

However, the environment 10 (50\%) was not supportive of it. Medical professionals were reported to be most influential on the alcoholic's decision to practice optimal dietary intake, although alcoholics' were almost anonymous in their belief that the final decision would be with them (Table 2). 
Citation: Mutuli LA, Bukhala P, Nguka G (2017) Factors Influencing Practice of Optimal Dietary Intake of Alcoholics under Rehabilitation in Asumbi-Homabay, Kenya. J Gen Pract (Los Angel) 5: 334. doi:10.4172/2329-9126.1000334

Page 4 of 7

\begin{tabular}{|c|c|c|}
\hline Normative beliefs & Frequency $(n=20)$ & Response percentage (\%) \\
\hline \multicolumn{3}{|l|}{ Balanced diet } \\
\hline \multicolumn{3}{|l|}{ Approval } \\
\hline Health professionals & 15 & 75 \\
\hline Family members & 12 & 62 \\
\hline Friends & 12 & 62 \\
\hline Colleagues & 10 & 50 \\
\hline \multicolumn{3}{|l|}{ Disapproval } \\
\hline Environment & 10 & 50 \\
\hline \multicolumn{3}{|c|}{ Eating a variety of foods } \\
\hline \multicolumn{3}{|l|}{ Approval } \\
\hline Health professionals & 12 & 62 \\
\hline Family members & 12 & 62 \\
\hline Friends & 10 & 50 \\
\hline \multicolumn{3}{|l|}{ Disapproval } \\
\hline Environment (others) & 15 & 75 \\
\hline Colleagues & 10 & 50 \\
\hline \multicolumn{3}{|l|}{ Diet adequacy } \\
\hline \multicolumn{3}{|l|}{ Approval } \\
\hline Health professionals & 10 & 50 \\
\hline Family members & 12 & 62 \\
\hline Friends & 7 & 37 \\
\hline Colleagues & 7 & 37 \\
\hline \multicolumn{3}{|l|}{ Disapproval } \\
\hline Environment (others) & 10 & 50 \\
\hline
\end{tabular}

Table 2: Normative beliefs affecting optimal dietary intake in alcohol rehabilitation.

\section{Control beliefs affecting optimal dietary intake}

Beliefs that may encourage/discourage optimal dietary intake as described by the practice of eating a balanced diet from variety of foods and consuming a diet that is adequate were subsequently reported. Participants reported the facilitators of eating a balanced diet to include 'nutrition knowledge in alcoholism' $15(75 \%)$ and 'the will power to recover from alcoholism' 12 (62\%). The barriers of balanced diet consumption were 'unavailability of foods' 17 (87\%) and 'inaccessibility to services of a nutritionist' 17 (87\%). Facilitators of eating variety of foods in diets during rehabilitation included 'nutrition knowledge in alcohol rehabilitation' 15 (75\%), and 'alcoholics health' $12(62 \%)$.

The participants also reported inaccessibility to services of a nutritionist' 17 (87\%) and 'unavailability of diverse foods' 15 (75\%) as a significant inhibitors of consumption of diverse foods during alcohol rehabilitation since they only ate similar diets served to them and had no alternatives. It was acknowledged that "time to prepare the food' 7 (37\%) was the least inhibitor of consumption of diverse foods since they consumed foods prepared by the kitchen section and were not allowed to cook for themselves.

Participant also reported 'influences from mass media' 10 (50\%), 'perceived misconception' 10 (50\%), 'people to prepare the foods' as other inhibitors of diverse food consumption. Participants reported 'accessibility to sufficient diets' $15(75 \%)$ as a significant facilitator of diet adequacy even though not diverse. The least facilitator of diet adequacy was 'alcoholic's nutrition status' reported by 10 (50\%) of the participants (Table 3). 
Citation: Mutuli LA, Bukhala P, Nguka G (2017) Factors Influencing Practice of Optimal Dietary Intake of Alcoholics under Rehabilitation in Asumbi-Homabay, Kenya. J Gen Pract (Los Angel) 5: 334. doi:10.4172/2329-9126.1000334

Page 5 of 7

\begin{tabular}{|c|c|c|}
\hline Control Factors & Response percentage (\%) & Frequency $(n=20)$ \\
\hline \multicolumn{3}{|l|}{ Eating a balanced diet } \\
\hline \multicolumn{3}{|l|}{ Facilitators } \\
\hline Nutrition knowledge in alcoholism & 75 & 15 \\
\hline Will power to recover from alcoholism & 62 & 12 \\
\hline \multicolumn{3}{|l|}{ Barriers } \\
\hline Unavailability of foods & 87 & 17 \\
\hline Inaccessibility to services of a nutritionist & 87 & 17 \\
\hline \multicolumn{3}{|l|}{ Eating a variety of foods } \\
\hline \multicolumn{3}{|l|}{ Facilitators } \\
\hline Nutrition knowledge in alcoholism & 75 & 15 \\
\hline Alcoholic's health after the period of alcoholism & 62 & 12 \\
\hline Confidence & 50 & 10 \\
\hline \multicolumn{3}{|l|}{ Barriers } \\
\hline Inaccessibility to services of a nutritionist & 87 & 17 \\
\hline Inaccessibility to variety of foods & 75 & 15 \\
\hline People to prepare the foods & 62 & 12 \\
\hline Time to prepare & 37 & 7 \\
\hline Misconceptions & 50 & 10 \\
\hline Wrong influences from mass media & 50 & 10 \\
\hline \multicolumn{3}{|l|}{ Diet adequacy } \\
\hline \multicolumn{3}{|l|}{ Facilitators } \\
\hline Nutrition Knowledge in alcoholism & 62 & 12 \\
\hline Alcoholic's nutrition status & 50 & 10 \\
\hline Supportive nutrition services & 62 & 12 \\
\hline Accessibility to sufficient diets & 75 & 15 \\
\hline \multicolumn{3}{|l|}{ Barriers } \\
\hline Lack of nutrition services & 87 & 17 \\
\hline Lack of capacity to quantify adequacy & 75 & 15 \\
\hline Poor appetite for food & 62 & 12 \\
\hline
\end{tabular}

Table 3: Control beliefs affecting optimal dietary intake in alcohol rehabilitation.

It was noted that familiarization with individual nutrition status was not of importance to the alcoholics. 'Lack of nutrition services' 17 (87\%) was mentioned as a major hindrance to sufficient diet with 15 (75\%) reporting the 'lack of capacity to quantify adequacy' as a barrier. 'Poor appetite for food' was also reported by $12(62 \%)$ of the participants as hindering their diet adequacy.
The information obtained from this coding phase was then organized into a coding paradigm that presents a framework of the process under investigation. In this way an attempt was made to build up a framework (Figure 1). 


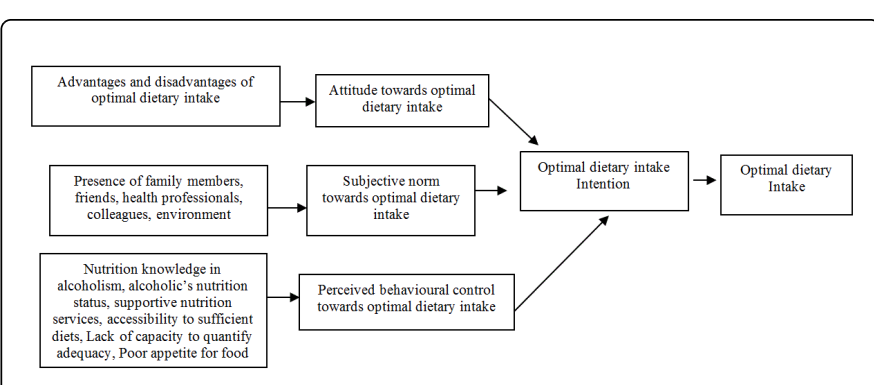

Figure 1: Measures of attitude, subjective norm and perceived behavioral control in optimal dietary intake.

\section{Discussion}

The recuperating alcoholics placed a very high value on the health benefits of practicing optimal dietary intake with intention of restoring their health which was previously impaired by the effect of alcoholism. Eating a balanced diet, diet variety and diet adequacy were reported as the channel towards optimal of dietary intake. There was certainty that eating balanced diets 'restored tissues, organs and body systems' which were impaired by the effect of alcoholism. Similar findings have been reported in previous studies examining the role of optimal dietary intake in alcoholism treatment $[9,10]$. 'Reducing alcohol craving, stabilizing neurotransmitters, avoidance of erratic blood sugars' could be achieved by adhering to diet variety. This concurred with some authors who emphasized the importance of eating varied diets during alcohol rehabilitation $[9,11]$. Accordingly, few authors argued that optimal diets should adequately meet the needs of a recuperating alcoholic correspondingly reducing nutrient deficiency and hydrating the body as reported in this study $[12,13]$. As illustrated in Table 1, there were fewer hindrances for practicing optimal dietary intake inclusive of the 'necessity of some skills and knowledge in nutrition', 'optimal diets were expensive' in terms of accessing and preparation. Attitudinal beliefs were perceived as the advantages and disadvantages of optimal dietary intake during alcohol rehabilitation. Majority of participants acknowledged the importance of optimal diet in enhancing their alcohol treatment at the rehab center even though this required adequate knowledge and skills in nutrition or assistance from a nutritionist. Normative influences emerged from health professionals, family members, partners, colleagues and friends since they were the people who had significant influence on optimal dietary intake intention of the recuperating alcoholics. The influences from these significant referents did show to affect the participant's decision on practise of optimal dietary intake during alcohol rehabilitation as displayed in Table 2. It suggested that a potential way to improve alcoholic's dietary intake is to intervene in the social norms that govern eating behaviour [13]. This qualitative analysis interpreted health professionals as being the major source of support regarding respondent's intention to practise optimal dietary intake as consistent with De Bruin [14]. This has essential implications for clinical practice of health professionals, who should capitalize on this finding to encourage alcoholics on treatment to initiate optimal dietary intake during the rehabilitation and follow up to ensure they maintain it even after discharge from the centre. However, previous studies have affirmed that follow up is rarely done especially after discharge from the centre. This may be a contributing factor to the prevalence of relapse after discharge from the rehabilitation centre. Social norms are the behavioral standards that exist in a social group for what is considered correct and appropriate behavior, and they emerge from the shared practices and expectations of the group members. When social and environmental support systems are in place, making healthful choices becomes possible and has an opportunity to improve alcohol rehabilitation. Perceived behavioral control represents participant's perceptions of control over practice of optimal dietary intake in the face of internal and external barriers; and the self-efficacy i.e., confidence that the participants have the ability to practice optimal dietary intake in spite of obstacles. It's essential for the recuperating alcoholics to receive individualized nutrition services from a nutritionist so that to improve their state of health and facilitate complete recovery from alcoholism. It's surprising that even though the recuperating alcoholics needed the support of a nutritionist, they had background information on role of nutrition in alcoholism. However, the complexity was to apply the knowledge into practise. This implies that they had low self-efficacy of practising optimal dietary intake as even illustrated in Table 3. This necessitates for a focus on rebuilding self-efficacy by creating realistic nutrition goals for participants [15]. On realization of the stated goals, participants should be offered affirmations for their accomplishments, being reminded that optimal dietary intake is possible regardless of present circumstances and despite previous track record.

\section{Conclusion}

This qualitative study established that deficiency of nutrition services was a significant barrier to the practise of optimal dietary intake during alcohol rehabilitation. This ascertains that scarcity of nutrition services leaves the recuperating alcoholics with mixed perceptions of the composition of an optimal diet especially for their condition. Fostering strong combined relationships amongst family members, health professionals, friends, colleagues and environment is fundamental to support an alcoholic on recuperation. The rehabilitation centres should have nutritionists besides other health professionals who can offer individualized nutrition services and support needed by the recuperating alcoholics. Increased self-efficacy in relation to optimal dietary intake may translate into increased selfefficacy regarding to practice of optimal dietary intake during alcohol rehabilitation. Optimal dietary intake alone is insufficient to keep an alcoholic sober, and self-reported perceptions of self-efficacy may not predict long-term abstinence. Optimal dietary intake in alcohol rehabilitation is a pathway that reflects self-care, commitment to staying sober and is a vital adjunct in the complete recovery from alcoholism and reduction of relapse. This may decrease the prevalence of relapse after discharge from the rehabilitation centre.

\section{Competing Interests}

The authors declare that no conflict of interests exists.

\section{Authors' Contributions}

All authors were involved with the drafting of the research paper, critically reviewed the manuscript and approved the final version submitted for publication.

\section{Acknowledgments}

The authors sincerely thank participants who shared their experiences, and contributed needed information to the study. All 
Citation: Mutuli LA, Bukhala P, Nguka G (2017) Factors Influencing Practice of Optimal Dietary Intake of Alcoholics under Rehabilitation in Asumbi-Homabay, Kenya. J Gen Pract (Los Angel) 5: 334. doi:10.4172/2329-9126.1000334

Page 7 of 7

those contributed to the success of this study in one way or another are also recognized.

\section{References}

1. Mathew RJ (2016) Eating to prevent alcohol cravings and relapse. Twelve Wellness.

2. Svanberg J, Evans JJ (2013) Neuropsychological rehabilitation in alcoholrelated brain damage: A systematic review. Alcohol Alcohol 48: 704-711.

3. Chesang RK (2013) Drug abuse among the youth in Kenya. Int J Sci Technol Res 2: 2277-8616.

4. Kuria MW (2013) Factors associated with relapse and remission of alcohol dependent persons after community based treatment. Open J Psychiatr 3: 264-272.

5. Chepkwony SJ, Chelule E, Barmao AC (2013) An investigation into prevalence and factors contributing to relapse among alcoholics in selected rehabilitation centres in Nairobi county, Kenya. Int J Innov Res Develop 2: 340-347.

6. Kahuthia-Gathu R, Okwarah P, Gakunju R, Thungu J (2013) Trends and emerging drugs in Kenya: A case study in Mombasa and Nairobi county. J App Biosci 67: 5308-5325.

7. Langat E, Wafula S, Ettyang G, Rotich J, Chelimo J (2014) The effect of rehabilitation on nutritional status of alcohol dependent males in Nairobi, Kenya. J Biol Agri Health 4: 85-101.
8. Ajzen I (1991) The theory of planned behavior, organizational behavior and human decision process. J Appl Soc Psychol 50: 109-119.

9. Maillot F, Farad S, Lamisse F (2001) Alcohol and nutrition. Pathol Biol (Paris) 4: 683.

10. McEachan RRC, Conner M, Taylor NJ, Lawton RJ (2011) Prospective prediction of health-related behaviours with the theory of planned behaviour: A meta-analysis. Health Psychol Rev 5: 97-144.

11. Griffith CM, Schenker S (2006) The role of nutritional therapy in alcoholic liver disease. Alcohol Res Health 29: 296-306.

12. Zakhari S, Li TK (2007) Determinants of alcohol use and abuse: Impact of quantity and frequency patterns on liver disease. Hepatology 46:2032-2039.

13. Moore JB, Gunn PJ, Fielding BA (2014) The role of dietary sugars and de novo lipogenesis in non-alcoholic fatty liver disease. Nutrients 6: 5679-5703.

14. De Bruin GJ, Kremers SPJ, Schaalma H, Van Mechelen W, Brug J (2005) Determinants of adolescent bicycle use for transportation and snacking behavior. Preventive Medicine 40: 658-667.

15. Dyson J (2007) Experiences of alcohol dependence: A qualitative study. J Fam Health Care 17: 211-214. 